\title{
DETERMINAÇÃO DE PARÂMETROS FÍSICO-QUÍMICOS E DA ORIGEM BOTÂNICA DE MÉIS INDICADOS MONOFLORAIS DO SUDESTE DO BRASIL'
}

\author{
Monika O. BARTH", Camila MAIORINO’, Ana P.T. BENATTI, Deborah H. M. BASTOS
}

\section{RESUMO}

O presente trabalho analisou a composição fisico-química e origem botânica de 31 amostras de mel indicadas como sendo de origem monofloral, comercializadas e produzidas na região Sudeste do Brasil, a fim de verificar parâmetros de qualidade. As análises físico-químicas compreenderam o teste de Fiehe, a reação de lugol, a determinação do teor de umidade, do pH, de açúcares redutores, de cinzas e do índice de diastase. Todas as amostras apresentaram-se dentro do limite previsto pela legislação brasileira para o teor de umidade. Dez amostras apresentaram teor de açúcares redutores inferior ao previsto na legislação, três tinham número de diastase (unidades Schade/Gothe) inferior a 8, uma apresentou pH abaixo do padrão e outra apresentou teor de cinzas superior ao previsto. A análise polinica mostrou que cerca de $57 \%$ das amostras poderiam ser classificadas como monoflorais, correspondendo nove amostras a mel de eucalipto (Eucalyptus, Myrtaceae), duas a mel de aroeira (Schinus, Anacardiaceae), duas a mel de assa-peixe (Vernonia, Asteraceae), duas a mel de laranjeira (Citrus, Rutaceae), uma a mel de cambará (Gochnatia, Asteraceae) e uma a mel de capixingui (Croton, Euphorbiaceae). As demais amostras eram bi- ou heteroflorais. A influência de uma determinada espécie botânica nas variáveis físico-quimicas analisadas não foi significativa $(p>0,05)$ segundo a análise de regressão.

Palavras-chave: mel; rotulagem; análise físico-química; análise polínica; origem botânica.

\section{SUMMARY}

PHYSICO-CHEMICAL PARAMETERS AND BOTANICAL ORIGIN OF INDICATED MONOFLORAL HONEYS FROM THE SOUTHEAST OF BRAZIL. The physico-chemical properties and the botanical origin of 31 commercial honey samples from Southeast Brazil, indicated as monofloral honeys, were analyzed in order to verify quality parameters. The physico-chemical analyses comprised the test of Fiehe, the lugol reaction, the determination of humidity, pH, reduced sugars, ashes and diastase. In accordance with Brazilian legislation all samples showed adequate water content. The level of reducing sugars from 10 samples was below the Brazilian quality parameters for honey, three presented a Schade/Gothe number of diastase activity below 8 and in one sample only a higher ash content. Pollen analysis showed that nearly $57 \%$ of the samples analyzed could be classified as monofloral honeys, corresponding to nine samples of Eucalyptus (Myrtaceae), two of Citrus (Rutaceae), two of Schinus (Anacardiaceae), two of Vernonia (Asteraceae), one of Croton (Euphorbiaceae) and one of Gochnatia (Asteraceae). The other samples were of bifloral or heterofloral origin. No relation between the floral origin and the physico-chemical properties of the samples examined could be detected using regression analysis $(\mathrm{p}>0.05)$.

Keywords: honey; label; physico-chemical analysis; pollen analysis; botanical origin.

\section{1 - INTRODUÇÃo}

Os parâmetros físico-químicos para méis brasileiros estão bem definidos [8]. Os principais componentes do mel são os açúcares, dos quais os monossacarídeos frutose e glicose perfazem cerca de $70 \%$; dissacarídeos, incluindo sacarose, somam cerca de $10 \%$ e a água de 17 a $20 \%[10,21,25]$. Muitas das características pelas quais o mel é apreciado, como o seu aroma e sabor, são determinadas por substâncias presentes em diminutas concentrações $[6,7]$.

Recebido para publicação em 29/07/2002. Aceito para publicação em 04/05/2005 (000947).

Laboratório de Palinologia, Departamentos de Botânica e de Geologia, Universidade Federal do Rio de Janeiro e Departamento de Virologia, Instituto Oswaldo Cruz, FIOCRUZ, Avenida Brasil, 4365, CEP: 21045-900, Rio de Janeiro-RJ, Brasil. E-mail: barth@ioc.fiocruz.br

Curso de Farmácia, Universidade São Francisco de Assis. CEP: 12900-000, Bragança Paulista-SP.

Departamento de Nutrição, Faculdade de Saúde Pública, Universidade de São Paulo, Av. Dr. Arnaldo, 715. CEP: 01246-904, São Paulo,Brasil.E-mail:dmbastos@usp.br

A quem a correspondência deve ser enviada.
Análises físico-químicas de méis brasileiros de abelhas africanizadas (Apis mellifera L.) têm sido a temática de algumas pesquisas. Uma extensa revisão sobre os glicídeos em mel brasileiro foi apresentada por MOREIRA \& DE MARIA [20]. A análise de 17 méis comerciais adquiridos na cidade de São Paulo [24] revelou que, exceto uma, as demais se encontravam dentro das normas estabelecidas pela legislação brasileira [8]. HORN [16] examinou 57 amostras de mel procedentes das regiões Sul, Sudeste, Norte e Nordeste do Brasil, encontrando variância significativa para os valores de $\mathrm{pH}$, umidade e lactonas, enquanto que para os valores de acidez total, hidroximetilfurfural e invertase as diferenças encontradas para estas quatro regiões não foram significativas.

A análise polínica, exclusivamente de méis monoflorais colhidos no Brasil, foi realizada por BARTH [2, 3] e BARTH \& CORÉ-GUEDES [4]. Diversos autores caracterizaram algumas amostras de mel monofloral dentro de um conjunto de amostras de procedência mais regional. Vale ressaltar os estudos de BASTOS et al. [5] no estado de Minas Gerais, CARREIRA \& JARDIM [12] no Pará, DUTRA \& BARTH [15] no estado do Rio de Janeiro e RAMALHO et al. [23] no Sul do Brasil.

Quanto às análises químicas de méis brasileiros rela- 
cionadas à origem botânica através do pólen, COSTA et al. [13] e DA COSTA LEITE et al. [14] examinaram 74 amostras de mel provenientes de 14 estados do Brasil, das quais 18 eram de mel monofloral de eucalipto ( $E u$ calyptus, Myrtaceae). Verificou-se que 18\% das amostras estavam fora dos padrões legais. Algumas amostras coletadas na região Nordeste mostraram índice de diastase surpreendentemente alto, devido, supostamente, à proximidade de estabelecimentos produtores de farinha de mandioca; outras, colhidas na região Centro-Oeste apresentaram teor de HMF relativamente elevado, mas ainda dentro dos padrões brasileiros.

Fato semelhante ocorreu quanto à origem floral de amostras de mel do Brasil examinadas por HORN [16], quando somente $10 \%$ do total de amostras analisadas correspondiam às informações das etiquetas.

Trinta e seis amostras de mel do estado de Minas Gerais foram analisadas quanto a parâmetros físicoquímicos e contribuição florística por BASTOS et al. [5]. A análise físico-química confirmou a presença de melato em alguns destes méis. Os autores verificaram que o mel de eucalipto, caracterizado por apresentar valores inferiores de diastase em relação a outros méis quando produzido durante a estação de seca naquela região, diferia do produzido na Espanha e na Austrália em função de valor mais elevado do $\mathrm{pH}$, do teor de nitrogênio e do teor mais baixo de frutose.

Adulterações do mel de abelhas pela adição de produtos açucarados, por superaquecimento ou produção de "mel análogo" ("high fructose corn syroup") são as mais freqüentes.

O presente trabalho tem como objetivo analisar a composição físico-química e palinológica de méis considerados monoflorais, produzidos na região Sudeste do Brasil, a fim de verificar e avaliar os dados obtidos em relação aos parâmetros legais.

\section{2 - MATERIAL E MÉTODOS}

\section{1 - Material}

Trinta e uma amostras de mel provenientes dos estados de São Paulo e Minas Gerais foram adquiridas em entrepostos comerciais ou obtidas diretamente dos apicultores. As amostras encontravam-se envasadas em frascos de vidro de $450 \mathrm{~g}$ ou $850 \mathrm{~g}$ e foram armazenadas a temperatura ambiente até análise. Todas as amostras foram analisadas dentro do prazo de validade constante do rótulo.

\section{2 - Métodos}

\subsection{1 - Análises fisico-químicas e de composição}

Teste de Fiehe - Realizado através de teste qualitativo para o hidroximetilfurfural de acordo com as normas analiticas do INSTITUTO ADOLFO LUTZ [17].

Reação de lugol - Este teste foi realizado com base na reação da solução de lugol, de acordo com as normas analíticas do INSTITUTO ADOLFO LUTZ [17].

Teor de umidade - O teor de umidade foi realizada por refratometria com interpretação através da tabela de Chatway, conforme descrito por BOGDANOV et al. [8].

$\mathrm{pH}-\mathrm{O}$ valor do $\mathrm{pH}$ foi obtido por potenciometria usando do potenciômetro Orion, 420 A, conforme descrito por BOGDANOV et al. [9]. A determinação foi feita em triplicata.

Teor de açúcares redutores - A determinação de açúcares redutores foi feita através de titulação de óxidoredução empregando-se o método de Felhing [9]. A determinação foi feita em triplicata.

Teor de cinzas - O teor de cinzas foi obtido por calcinação das amostras de mel, a $600^{\circ} \mathrm{C}$, em mufla, até a obtenção de uma massa constante [9]. A determinação foi feita em triplicata.

Índice de diastase - O índice de diastase foi obtido pelo método descrito por BOGDANOV et al. [9], expressso em unidades Schade ou Gothe por grama de mel. A regressão linear foi calculada utilizando-se a calculadora científica HP 12C. A determinação foi feita em duplicata.

\subsection{2 - Análise polínica}

O preparo das amostras, baseado no conteúdo polínico de $10 \mathrm{~g}$ de mel, seguiu o método padronizado europeu, sem aplicação de acetólise [1, 19]. A identificação dos tipos polínicos baseou-se na coleção de referência do Laboratório de Palinologia, Departamento de Botânica, Instituto de Biologia, Universidade Federal do Rio de Janeiro.

\subsection{3 - Análise de regressão múltipla}

Utilizando-se o pacote estatístico SPSS efetuou-se análise de regressão múltipla para verificar se os parâmetros físico-químicos poderiam ser utilizados para classificar a amostra em função da origem floral. Nesse caso, considerou-se variável dependente a origem floral e variáveis independentes os parâmetros físico-químicos.

\section{3 - RESULTADOS E DISCUSSÃo}

Os dados obtidos estão apresentados na Tabela 1 quanto aos parâmetros físico-químicos das amostras. O teor de água variou de 15 a $20 \%$, o de açúcares redutores de 67,4 a $83,2 \%$ e o de dissacarídeos e oligossacarídeos de zero a 23\%. Quando possível de ser determinado, o número de diastase variou de 5 a 19 unidades Schade. Ovalor de $\mathrm{pH}$ oscila entre 3,0 e 4,3 e o do teor de cinzas entre 0,03 e $0,88 \%$.

Todas as amostras analisadas apresentaram resultados negativos para o teste de Fiehe e a reação de lugol, indicando que não foram submetidas a condições severas de aquecimento, nem sofreram adição de xaropes açucarados.

O teor de umidade dos méis analisados variando en- 
tre $15 \%$ e $20 \%$, ficou dentro do padrão, correspondente ao máximo permitido de $20 \%$.

A concentração mínima de açúcares redutores, segundo a legislação brasileira é de $72 \%$. De acordo com a regulamentação vigente, as amostras $1,9,10,17-20$ e 25-27 ficaram abaixo do limite admissível. Entre estas amostras, cinco (amostras 10, 17, 20, 25 e 26) são procedentes de florada de assa-peixe e cambará, quatro contem néctar de eucalipto (amostras 9, 18, 19, 27) e duas apresentaram uma parte extrafloral (amostras 9, 27). Esses resultados ressaltam a necessidade de conhecer melhor a sua composição de açúcares. Entretanto, as amostras de méis monoflorais de eucalipto (amostras 5$7,14,15,23,30$ ), exceto a amostra 18, encontram-se dentro do padrão estabelecido.

A Tabela 1 apresenta uma estimativa do teor de dissacarídeos e oligissacarídeos obtida por diferença. Optamos por utilizar esta estimativa visto que as amostras foram armazenadas a temperatura ambiente por intervalo de tempo variável (para cada amostra) e visto que a literatura relata que o teor de dissacarídeos (principalmente sacarose) muda nestas condições, diminuindo drasticamente $[11,18]$. Assim, a análise (quantitativa) do teor de açúcares totais presentes nas amostras, pelo método de Fehling, traria uma informação que não poderia ser interpretada, dado às modificações que podem ter ocorrido e às próprias condições de análise [22]. Estando o limite estabelecido para açúcares não redutores em sacarose em no máximo $10 \%$, diversas amostras aqui analisadas o ultrapassaram (Tabela 1).

Os valores médios de $\mathrm{pH}$, pela legislação brasileira, oscilam entre 3,3 e 4,6; somente a amostra 7 está abaixo dos limites fixados.

O teor de cinzas permitido, de acordo com a legislação brasileira, pode chegar até $0,6 \%$. Todas as amostras, exceto a amostra 3, estão dentro dos limites fixados. A amostra 3 é um mel escuro de laranjeira com melato, sendo que o valor elevado de cinzas é provavelmente devido à parte do melato.

A Comunidade Européia e o Codex Alimentaris estabelecem que o mel de abelhas, após o processamento, não deve apresentar um número de diastase inferior a 8 unidades Schade (Gothe). No entanto, para méis que apresentam baixo teor de enzimas, esse limite pode estender-se ate 3 unidades Schade. Das amostras analisadas três $(8,22,23)$ apresentaram número inferior a 8. Para 18 amostras não foi determinado o índice de diastase, pois a absorbância inicial estava abaixo do previsto na metodologia (resultados descritos como $n$ na Tabela 1). Esse resultado indica que ou as diastases presentes (amilases $\alpha$ e $\beta$ ) possuem uma atividade muito alta, ou a presença de outras enzimas ou outros fatores estão interferindo nesta análise.

Em relação aos estudos realizados sobre as propriedades fisico-químicas de mel de abelhas do Brasil, constatou-se irregularidades quanto ao cumprimento da le- gislação brasileira em amostras de mel provenientes de várias regiões do território nacional. Assim, valores elevados de diastase foram constatados por COSTA et al. [13] para a região Nordeste, enquanto que as examinadas no presente trabalho, região Sudeste, apresentaram muitas vezes valores abaixo de 3 unidades Schade. Já em 17 amostras de São Paulo [24] somente uma estava inteiramente fora dos padrões e 6 também apresentaram valores de Schade abaixo de 8 .

Quanto à origem botânica (Tabela 2) puderam ser identificadas 17 amostras como méis monoflorais, sendo nove de eucalipto (Eucalyptus, Myrtaceae), dois de aroeira (Schinus, Anacardiaceae), dois de assa-peixe (Vernonia, Asteraceae), dois de laranjeira (Citrus, Rutaceae) e uma repectivamente de cambará (Gochnatia, Asteraceae) e capixingui (Croton, Euphorbiaceae). Cinco amostras são méis biflorais e 8 apresentam mais de dois taxa botâ-

TABELA 1- Parâmetros físico-quimicos e de composição das amostras de mel analisadas

\begin{tabular}{|c|c|c|c|c|c|c|}
\hline Amostra $n^{\circ}$ & $\begin{array}{c}\text { Teor de água em } \\
\%\end{array}$ & $\begin{array}{c}\text { "Teor de } \\
\text { açucares } \\
\text { redutores } \\
\%\end{array}$ & $\begin{array}{c}\text { "*Teor de } \\
\text { dissacarideos e } \\
\text { digossacarideos } \\
(\%)\end{array}$ & $\begin{array}{l}\text { *k\#Número de } \\
\text { diastase (Unidades } \\
\text { Shade) }\end{array}$ & $\mathrm{pH}$ & $\begin{array}{c}{ }^{\text {*Teor de Cinzas }} \\
\%\end{array}$ \\
\hline 1 & 16,8 & 69,9 & 13 & $n$ & 3,4 & 0,10 \\
\hline & & $(0,38)$ & & & $(0,01)$ & $(0,02)$ \\
\hline 2 & 15,0 & $\begin{array}{r}81,7 \\
(0,47)\end{array}$ & 3,3 & $\mathrm{n}$ & $\begin{array}{c}3,8 \\
(0,01)\end{array}$ & $\begin{array}{l}0,16 \\
(0,15)\end{array}$ \\
\hline 3 & 16,1 & 72,0 & 12 & $\mathrm{n}$ & 3,6 & 0,88 \\
\hline 4 & 19,2 & $\begin{array}{r}0,66) \\
74,6\end{array}$ & 6,2 & $n$ & $\begin{array}{c}(0,01) \\
3,9\end{array}$ & $\begin{array}{c}(0,0) \\
0,07\end{array}$ \\
\hline 5 & 15,2 & $\begin{array}{l}(0,47) \\
79,1\end{array}$ & 5,7 & $n$ & $\begin{array}{l}(0,06) \\
3,6\end{array}$ & $\begin{array}{l}(0,01) \\
0,15\end{array}$ \\
\hline 6 & 19,0 & $\begin{array}{r}(1,62) \\
74,9 \\
(0,44)\end{array}$ & 6,1 & $\mathrm{n}$ & $\begin{array}{c}(0,01) \\
3,5 \\
(0,03)\end{array}$ & $\begin{array}{l}(0,02) \\
0,16 \\
(0,03)\end{array}$ \\
\hline 7 & 18,6 & $\begin{array}{r}79,8 \\
(0,25)\end{array}$ & 1,6 & $\mathrm{n}$ & $\begin{array}{r}3,0 \\
(0,01)\end{array}$ & $\begin{array}{l}0,13 \\
(0,02)\end{array}$ \\
\hline 8 & 16,2 & $\begin{array}{r}75,5 \\
10500\end{array}$ & 8,3 & 7 & 3,6 & $\begin{array}{l}0,25 \\
0,25\end{array}$ \\
\hline 9 & 17,2 & $\begin{array}{l}(U, 50) \\
71,8\end{array}$ & 11 & $\begin{array}{c}(0,4) \\
17\end{array}$ & $\begin{array}{c}(0,26) \\
3,9\end{array}$ & $\begin{array}{c}(0,25) \\
0,20\end{array}$ \\
\hline 10 & 17,2 & $\begin{array}{l}(1,62) \\
69,8\end{array}$ & 13 & $\begin{array}{l}(0,6) \\
13\end{array}$ & $\begin{array}{l}(0,72) \\
3,5\end{array}$ & $\begin{array}{l}(0,03) \\
0,06\end{array}$ \\
\hline 11 & 16,8 & $\begin{array}{c}(0,25) \\
73,8 \\
(0,54)\end{array}$ & 9,4 & $\begin{array}{c}(0,3) \\
14 \\
(0,4)\end{array}$ & $\begin{array}{c}(0,01) \\
3,4 \\
(0,05)\end{array}$ & $\begin{array}{c}(0,03) \\
0,19 \\
(0,02)\end{array}$ \\
\hline 12 & 18,0 & $\begin{array}{r}74,9 \\
(0,44)\end{array}$ & 7,1 & $n$ & $\begin{array}{c}3,4 \\
(0,75)\end{array}$ & $\begin{array}{c}0,17 \\
(0,03)\end{array}$ \\
\hline 13 & 18,8 & 79,8 & 1,4 & $\mathrm{n}$ & 3,4 & 0,05 \\
\hline 14 & 17,8 & $\begin{array}{r}(0,25) \\
78,7\end{array}$ & 3,5 & 12 & $\begin{array}{c}(0,0) \\
3,6\end{array}$ & $\begin{array}{l}(0,06) \\
0,06\end{array}$ \\
\hline 15 & 18,4 & $\begin{array}{l}0,61) \\
79,8\end{array}$ & 1,8 & $\begin{array}{l}(0,2) \\
18\end{array}$ & $\begin{array}{c}(0,00) \\
3,7\end{array}$ & $\begin{array}{l}0,20) \\
0,2\end{array}$ \\
\hline 16 & 15,8 & $\begin{array}{l}(0,29) \\
73,7 \\
0,0)\end{array}$ & 10,5 & $\begin{array}{l}(0,1) \\
18\end{array}$ & $\begin{array}{l}(0,1,5) \\
3,5\end{array}$ & $\begin{array}{l}(0,08) \\
0,03\end{array}$ \\
\hline 17 & 16,6 & $\begin{array}{l}(0,20) \\
70,5\end{array}$ & 12,9 & $\begin{array}{l}(1,3) \\
19\end{array}$ & $\begin{array}{l}(0,0) \\
3,5\end{array}$ & 0,06 \\
\hline 18 & 18,4 & $\begin{array}{l}(0,33) \\
67,4\end{array}$ & 14,2 & $\stackrel{(0,9)}{n}$ & $\begin{array}{c}(0,03) \\
4,3\end{array}$ & $\begin{array}{l}(0,01) \\
0,23\end{array}$ \\
\hline 19 & 15,0 & $\begin{array}{l}(0,30) \\
70,7\end{array}$ & 14,3 & $n$ & $\begin{array}{r}(0,04) \\
3,7 \\
0\end{array}$ & $\begin{array}{l}(0,03) \\
0,05 \\
(0,03)\end{array}$ \\
\hline 20 & 17,4 & 68,6 & 14 & $n$ & $\begin{array}{l}3,7 \\
0\end{array}$ & 0,35 \\
\hline 21 & 17,2 & $\begin{array}{l}74,7 \\
\end{array}$ & 8,1 & 8 & 3,6 & 0,05 \\
\hline 22 & 19,8 & 76,8 & 3,4 & 5 & 3,3 & $\begin{array}{l}0,12 \\
0,12\end{array}$ \\
\hline 23 & 18,4 & $\begin{array}{c}(0,32) \\
83,2\end{array}$ & 0,0 & 13 & $\begin{array}{c}(0,04) \\
3,3\end{array}$ & $\begin{array}{c}0,05 \\
0,05\end{array}$ \\
\hline 24 & 19,0 & $\begin{array}{r}0,46) \\
78,4 \\
(0,41)\end{array}$ & 2,6 & $\begin{array}{c}(0,2) \\
6 \\
(0,2)\end{array}$ & $\begin{array}{c}(0,01) \\
3,4 \\
(0,02)\end{array}$ & $\begin{array}{l}(0,02) \\
0,13 \\
(0,04)\end{array}$ \\
\hline 25 & 16,2 & $\begin{array}{r}60,7 \\
(0,25\end{array}$ & 23 & $n$ & $\begin{array}{r}3,8 \\
0\end{array}$ & $\begin{array}{r}0,10 \\
0,01\end{array}$ \\
\hline 26 & 16,2 & $\begin{array}{r}62,0 \\
(0,69\end{array}$ & 21,8 & $\mathrm{n}$ & $\begin{array}{c}3,8 \\
(0,02)\end{array}$ & $\begin{array}{r}0,10 \\
(006)\end{array}$ \\
\hline 27 & 16,2 & $\begin{array}{r}63,6 \\
10299\end{array}$ & 20,2 & $\begin{array}{c}13 \\
(0,3)\end{array}$ & $\begin{array}{r}3,8 \\
(0,04)\end{array}$ & $\begin{array}{c}0,10 \\
(0,06)\end{array}$ \\
\hline 28 & 17,2 & $\begin{array}{c}74,1 \\
(0,00)\end{array}$ & 8,7 & $n$ & $\begin{array}{c}3,5 \\
(0,07\end{array}$ & $\begin{array}{l}0,07 \\
(0,01)\end{array}$ \\
\hline 29 & 17,4 & $\begin{array}{l}79,3 \\
(0,94)\end{array}$ & 3,3 & $n$ & $\begin{array}{c}3,6 \\
(0,02)\end{array}$ & $\begin{array}{l}0,21 \\
(0,04)\end{array}$ \\
\hline 30 & 20,0 & $\begin{array}{c}79,1 \\
(0,42)\end{array}$ & 0,9 & $\mathrm{n}$ & $\begin{array}{c}3,6 \\
(0,03)\end{array}$ & $\begin{array}{l}0,14 \\
0,04)\end{array}$ \\
\hline 31 & 16,6 & $\begin{array}{r}77,2 \\
(0,55)\end{array}$ & 6,2 & $\mathrm{n}$ & $\begin{array}{c}4,1 \\
(0,02) \\
\end{array}$ & $\begin{array}{r}0,15 \\
(0,06) \\
\end{array}$ \\
\hline
\end{tabular}

*média de triplicata. O número entre parênteses refere-se ao desvio padrão.

**Este valor foi obtido subtraindo-se a soma do teor de umidade mais o teor de açúcares redutores e subtraindo-se de 100 .

*** média de duplicata. O número entre parênteses refere-se ao desvio. $\mathrm{n}=$ para essas amostras, a absorbância obtida após a incubação da amostra com a solução de amido, nos primeiros 5 minutos, era inferior a 0,235 e portanto o número de diastase não foi determinado. 
TABELA 2 - Origem botânica das amostras analisadas conforme declaração nos rótulos e os resultados das análises polínicas

\begin{tabular}{|c|c|c|c|}
\hline Amostra $\mathrm{n}^{\circ}$ & Estado & $\begin{array}{l}\text { Origem botânica } \\
\text { descrita no rótulo }\end{array}$ & $\begin{array}{c}\text { Origem botânica determinada por análise } \\
\text { polínica }\end{array}$ \\
\hline 1 & $\mathrm{SP}$ & $\frac{\text { cipó-uva }}{\text { cou }}$ & Não determinado (sem material disponivel) \\
\hline 2 & SP & cipó-uva & $\begin{array}{l}\text { mel de laranjeira (Citrus, Rutaceae) com cipó- } \\
\text { uya (Cupania Sapindaceae) }\end{array}$ \\
\hline 3 & SP & cipó-uva & mel de laranjeira com melato \\
\hline 4 & $\mathrm{SP}$ & cipó-uva & mel de laranjeira e cipó-uva \\
\hline 5 & SP & capixingui & mel de eucalipto (Eucalyptus, Myrtaceae) \\
\hline 6 & SP & capixingui & mel de eucalipto e parte extrafloral \\
\hline 7 & SP & capixingui & mel de eucalipto e parte extrafloral \\
\hline 8 & $\mathrm{SP}$ & assa-peixe & $\begin{array}{l}\text { mel de cambará (Gochnatia, Asteraceae), } \\
\text { eucalipto e assa-peixe Vemonia, Asteraceae) }\end{array}$ \\
\hline 9 & $\mathrm{SP}$ & Assa-peixe & mel extrafloral com contribuiçẫo de eucalipto \\
\hline 10 & SP & assa-peixe & $\begin{array}{l}\text { mel de assa-peixe, cambará e margaridão } \\
\text { (Montanoa Asteraceae) }\end{array}$ \\
\hline 11 & SP & assa-peixe & mel de cambará \\
\hline 12 & $\mathrm{SP}$ & assa-peixe & mel de assa-peixe \\
\hline 13 & SP & assa-peixe & mel de eucalipto, cambará e assa-peixe \\
\hline 14 & $\mathrm{SP}$ & assa-peixe & mel de eucalipto \\
\hline 15 & SP & assa-peixe & mel de eucalipto \\
\hline 16 & MG & assa-peixe & mel de eucalipto, cambará e assa-peixe \\
\hline 17 & MG & assa-peixe & cambarà, assa-peixe e eucalipto \\
\hline 18 & MG & assa-peixe & mel de eucalipto \\
\hline 19 & MG & assa-peixe & mel de eucalipto com cambará \\
\hline 20 & MG & assa-peixe & eucalipto, cambará e assa-peixe \\
\hline 21 & SP & assa-peixe & mel de laranjeira \\
\hline 22 & SP & assa-peixe & mel de capixingui \\
\hline 23 & SP & assa-peixe & mel de eucalipto \\
\hline 24 & SP & assa-peixe & mel de assa-peixe e florada mista \\
\hline 25 & $\mathrm{SP}$ & assa-peixe & mel de assa-peixe \\
\hline 26 & SP & assa-peixe & mel de cambará, assa-peixe e eucalipto \\
\hline 27 & $\mathrm{SP}$ & assa-peixe & $\begin{array}{l}\text { eucalipto, erva-de-santa-cruz (Eupatorium, } \\
\text { Asteraceae) e extrafloral }\end{array}$ \\
\hline 28 & SP & cipó-uva & $\begin{array}{l}\text { mel de aroeira (Schinus, Anacardiaceae) e } \\
\text { cipó-uva }\end{array}$ \\
\hline 29 & SP & cipó-uva & mel de aroeira \\
\hline 30 & SP & capixingui & mel de eucalipto \\
\hline 31 & SP & cipó-uva & mel de aroeira \\
\hline
\end{tabular}

nicos. Melato foi identificado em uma amostra associado ao pólen de laranjeiras. Há contribuição de produtos extraflorais (não apresentam indicadores de melato) em quatro amostras de mel de eucalipto, uma das quais apresenta ainda pólen de erva-de-santa-cruz (Eupatorium, Asteraceae).

Segundo a Tabela 2, houve grande discordância entre a determinação da origem botânica das amostras de mel fornecidas pelos produtores e a obtida através da análise polínica. Houve concordância somente em duas amostras de assa-peixe (amostras 12, 24). Das 30 amostras recebidas como mel monofloral, somente cerca de $57 \%$ eram realmente procedentes de uma única espécie vegetal. Cinco amostras eram biflorais (cerca de 17\%) e cerca de $27 \%$ (8 amostras) eram heteroflorais. Melato foi encontrado em somente uma amostra. Já uma contribuição extrafloral foi constatada em 4 amostras (cerca de $13 \%)$. A falta de correspondência entre a verdadeira origem botânica de mel e a indicada pelo comércio confirma os resultados apresentados por COSTA et al. [13], DA COSTA LEITE et al. [14] e HORN [16].

\section{4 - CONCLUSÕES}

Não foi observado resultado significativo através da análise de regressão para verificar se a presença de uma determinada espécie floral influência significativamente nas variáveis físico-químicas analisadas. No entanto, a análise da correlação entre as variáveis estudadas mostra que o índice de diastase está correlacionado com o pH (nivel de 5\% de significância). A atividade enzimática depende efetivamente do $\mathrm{pH}$ do meio.

Os resultados obtidos mostram que, com alta freqüência, os méis comercializados como monoflorais indicam, incorretamente sua origem botânica. Assim, destaca-se a necessidade de conduzir a análise polínica para verificação da origem botânica dos méis. É necessário também compilar dados sobre a composição físicoquímica dos méis produzidos na região Sudeste do Brasil, pois há indícios de que os padrões estabelecidos pela legislação brasileira não contemplariam alguns desses méis. Uma parte das amostras analisadas não poderia ser classificada como mel de mesa em função do teor de açúcares redutores. O comportamento de várias amostras, relativo às enzimas amiloliticas, merece atenção e futuras investigações

\section{5 - REFERÊNCIAS BIBLIOGRÁFICA}

[1] BARTH, O.M.. O Pólen no Mel Brasileiro. Rio de Janeiro: Luxor, 1989.

[2] BARTH, O.M. Pollen in monofloral honeys from Brazil. Journal of Apicultural Research, v.28, p.89-94, 1990.

[3] BARTH, O.M. Monofloral and wild flower honey pollen spectra at Brazil. Ciência e Cultura, v. 48, p. 163-165, 1996.

[4] BARTH, O. M.; CORÉ-GUEDES, J. Caracterização de méis de laranjeiras procedentes dos estados do Rio de Janeiro e de São Paulo, por meio da análise polínica. LECTA, v. 17, p. 27-35, 1999.

[5] BASTOS, E.M; DAYRELL, I.O.; SAMPAIO, I.B.; JOLK, L. Correlation between pollen spectrum and physicochemical properties of honeys produced in the Minas Gerais state (Brazil). Ciênc. Tecnol. Aliment., v. 16, p. 146-152, 1996.

[6] BASTOS, D.H.M.; DA SILVA, M.A.A.P.; FRANCO, M.R.B. Otimização da etapa de isolamento dos compostos voláteis de mel para análise por cromatografia gasosa. Alimentos e Nutrição, v.9, p.77-88, 1998.

[7] BASTOS,D.H.M. FRANCO,M.R.B.; DA SILVA,M.A.A.P.; JANZANTI,N.S.; MARQUES, M.O.M. Composição de voláteis e perfil sensorial de méis de eucalipto e laranja produzidos nos Estados de São Paulo e de Minas GeraisCiênc. Tecnol. Aliment. , v.22, n.2,p.122-129, 2002.

[8] BRASIL, Ministério da Agricultura, Portaria SIPA n. 367 de 04 de setembro de 1997. Normas higiênico-sanitárias e tecnológicas para mel, cera de abelhas e derivados.

[9] BOGDANOV, S. LULLMANN, C.; MARTIN, P.; VON DER OHE, W.; RUSSMANN, H.; VORWOHL, G.;ODDO, 
L.P.;SABATINI, A.G.; MARCAZZAN, G.L.; PIRO, R.; FLAMINI, C; MORLOT, M.; LHÉRITIER, J.; BORNECK, R.; MARIOLEAS, P.; TSIGURI, A.; KERKVLIET, J.; ORTIZ, A.; IVANOV, T.; DARCY, B.; MOSSEL, B.; VIT, P. Honey quality and international regulatory standards: review by the International honey commission. Bee Word, v.80, n.2, p.61-69, 1999.

[10] CAMPOS, M. Contribuição para o estudo do mel, pólen, geléia real e própolis. Boletim da Faculdade de Farmácia, v. 11, p. 17-47, 1987.

[11] CHERCHI, A.; POREN, M.; SPANEDDA, L.; TUBEROSO, C.I.G. Influenza dell invecchiamento sulla qualitá del miele. Industria Conserve, v.73, p. 266 $271,1997$.

[12] CARREIRA, L.M.M.; JARDIM, M.A. Análise polinica dos méis de alguns municípios do Estado do Pará-II. Boletim do Museu Paraense Emilio Goeldi, Série Botânica, Belém, v.10, p. 83-89, 1994.

[13] COSTA, L.S.M.; ALBUQUERQUE, M.L.S.; TRUGO, L.C.; QUINTEIRO, L.M.C.; BARTH, O.M.; RIBEIRO, M.; DE MARIA,C.A.B. Determination of non-volatile compounds of different botanical origin brazilian honeys. Food Chemistry, v. 65, p. 347-352, 1999.

[14] DA COSTA LEITE,J.M.; TRUGO,L.C.; COSTA,L.S.M.; QUINTEIRO,L.M.C.; BARTH, O.M.; DUTRA, V.N.L.; DE MARIA, C.A.B.. Determination of oligosaccharides in Brazilian honeys of different botanical origin. Food Chemistry, v. 70, p. 93-98, 2000.

[15] DUTRA, V.M.L.; BARTH, O.M. Análise palinológica de amostras de mel da região de Bananal (SP/RJ), Brasil. Revista Universidade Guarulhos, Geociências II ( $\mathrm{n}^{-}$ especial), Guarulhos, p.174-183, 1997.

[16] HORN, H. Méis brasileiros: resultados de análises fisicoquímicas e palinológicas. Mensagem Doce, São Paulo, v. 40, p. 10-16, 1997.
[17] InStituTO ADOLFO LUTZ, SÃO PAUlO. Normas Analiticas do Instituto Adolfo Lutz, 3ed. São Paulo, v. 1, p. 21-2, 27-8, 42-3, 1985.

[18] JIMÉNEZ, M.; MATEO, J.J.; HUERTA, T.; MATEO, R. Influence of the storage conditions on some physicochemical and mycological parameters of honey. Journal of the Science of Food and Agriculture, v. 64, p. 67-74, 1994.

[19] LOUVEAUX, J.; MAURIZIO, A.; VORWOHL, G. Methods of melissopalinology. Bee World, v. 50, p. 139-157, 1978.

[20] MOREIRA, R.F.A; DE MARIA, C.A.B. Glicídios no mel. Química Nova, v. 24, p. 516-525, 2001.

[21] ODDO, L.P.; PIANA, L.; SABATINI, A.G. Conoscere il miele guida all'analisi sensoriale. 2 ed. Bolonha :Avenue Media , 1996.

[22] POMERANZ, Y.; MELOAN, C.E. Food Analysis: Theory and Practice. 3 edition. New York: Chapman \& Hall, 1994, p.625-677.

[23] RAMALHO, M.; GUIBU, L.S.; GIANNINI, T.C.; KLEINERTGIOVANNINI, A.; IMPERATRIZ-FONSECA, V.L. Characterization of some southern Brazilian honey and bee plants through pollen analysis. Journal of Apicultural Research, v.30, p.81-86, 1991.

[24] VILHENA, F.; ALMEIDA-MURADIAN, L.B. Análises físicoquímicas de méis de São Paulo. Mensagem Doce, São Paulo, v. 53, p. 17-19, 1999.

[25] WHITE, J.W. Honey. Advances in Food Research, v. 24, p. 287-374, 1978.

\section{6 - AGRADECIMENTOS}

Os autores agradecem à FAPESP pela bolsa concedida à autora Camila Maiorino (processo 98/11982-7)e ao CNPq pela bolsa concedida à primeira autora. 